\title{
HER2 copy number as predictor of disease-free survival in HER2-positive resectable gastric adenocarcinoma
}

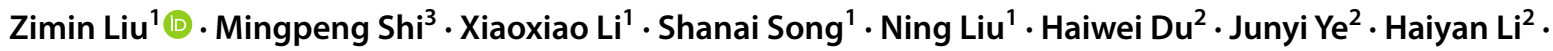 \\ Zhou Zhang $^{2} \cdot$ Lu Zhang ${ }^{2}$
}

Received: 2 August 2020 / Accepted: 10 January 2021 / Published online: 4 February 2021

(c) The Author(s) 2021

\begin{abstract}
Purpose The identification of HER 2 overexpression in a subset of gastric adenocarcinoma (GA) patients represents a significant step forward in unveiling the molecular complexity of this disease. The predictive and prognostic value of HER2 amplification in advanced HER2 inhibitor-treated GA patients has been investigated. However, its predictive value in resectable patients remains elusive.

Methods We enrolled 98 treatment-naïve resectable Chinese GA patients with HER2 overexpression assessed using IHC. Capture-based targeted sequencing using a panel consisting of 41 gastrointestinal cancer-related genes was performed on tumor tissues. Furthermore, we also investigated the correlation between HER2 copy number (CN) and survival outcomes. Results Of the 98 HER2-overexpressed patients, 90 had HER2 CN amplification assessed using next-generation sequencing, achieving 92\% concordance. The most commonly seen concurrent mutations were occurring in TP53, EGFR and PIK3CA. We found HER2 CN as a continuous variable was an independent predictor associated with DFS $(p=0.029)$. Our study revealed HER $2 \mathrm{CN}$-high patients showed a trend of intestinal-type GA predominant $(p=0.075)$ and older age $(p=0.07)$. The median HER2 CN was 15.34, which was used to divide the cohort into $\mathrm{CN}$-high and $\mathrm{CN}$-low groups. Patients with high HER2 CN had a significantly shorter DFS than patients with low HER2 CN $(p=0.002)$. Furthermore, HER2 $\mathrm{CN}$ as a categorical variable was also an independent predictor associated with DFS in patients.

Conclusion We elucidated the mutation spectrum of HER2-positive resectable Chinese GA patients and the association between HER2 CN and DFS. Our work revealed HER $2 \mathrm{CN}$ as an independent risk factor predicted unfavorable prognosis in HER2-positive GA patients and allowed us to further stratify HER2-positive resectable GA patients for disease management.
\end{abstract}

Keywords $H E R 2 \cdot$ Copy number $\cdot$ Resectable gastric adenocarcinoma $\cdot$ Survival

\section{Introduction}

Gastric cancer (GC) is the fifth most frequently diagnosed cancer and the third leading cause of cancer death in the world (Bray et al. 2018). Despite a worldwide decline in the incidence of GC, the mortality rate due to GC remains high (Siegel et al. 2020) because the majority of patients

Zimin Liu

liuzimin301@126.com

1 Oncology Department, The Affiliated Hospital of Qingdao University, Qingdao 266000, China

2 Burning Rock Biotech, Guangzhou, China

3 Operating Room of Neurosurgery, The Affiliated Hospital of Qingdao University, Qingdao 266000, China are diagnosed at an advanced stage and patients undergoing curative tumor resection for early-stage-GC frequently experience recurrence and or progression to metastatic disease (Hohenberger and Gretschel 2003; Van Cutsem et al. 2016).

$H E R 2$ (known as ERBB2, human epidermal growth factor receptor 2) represents the first molecular target for GC patients. It encodes human epidermal growth factor receptor 2, a $185 \mathrm{KD}$ transmembrane glycoprotein receptor with intracellular tyrosine kinase activity, and is activated by homodimerization or heterodimerization with other ERBB receptors including HER3 (Coussens et al. 1985; Hsieh and Moasser 2007; King et al. 1985; Ma et al. 2014). It is well known that $8-30 \%$ gastric adenocarcinomas (GA) show HER 2 overexpression or gene amplification (Aditi et al. 2016; Bang et al. 2010; Cordero-García et al. 2019; Qiu et al. 2017; Shen et al. 2016; Shitara et al. 2013). 
Overexpression of HER2 drives tumorigenesis, resulting in activated oncogenic downstream signaling, such as $\mathrm{PI} 3 \mathrm{~K} / \mathrm{Akt} / \mathrm{mTOR}$ and MAPK, promoting cellular proliferation, survival and angiogenesis in vitro and in vivo (Hynes and Stern 1994; Moasser 2007; Yarden and Sliwkowski 2001). Based on that observation, HER 2 has been targeted for cancer treatment.

Several studies reported that HER2 overexpression predicts unfavorable prognosis in unresectable GC patients (Mizutani et al. 1993). HER2 is also a predictive marker for survival benefit in advanced GC patients treated with trastuzumab plus chemotherapy. The ToGA (Trastuzumab for Gastric Cancer) trial reveals that HER2- positive patients experienced longer overall survival (OS) after treated with trastuzumab plus chemotherapy compared with HER2-positive patients treated with chemotherapy alone (Bang et al. 2010). However, the prognostic value of HER2 overexpression or gene amplification in resectable GA patients remains controversial. Some studies reported HER2 overexpression predicts unfavorable prognosis (Garcia et al. 2003; Kurokawa et al. 2015), but other studies failed to find an association between HER2 expression level and prognosis (Kim et al. 2019; Shen et al. 2016). In the present work, HER2 amplification was assessed by targeted next-generation sequencing (NGS) using a panel consisting of 41 gastrointestinal cancer-related genes, and the predictive and prognostic value of $H E R 2$ amplification in patients with HER2-positive resectable GA was investigated.

\section{Methods}

\section{Patients and specimens}

A total of 98 consecutive patients with any stage of primary GA and positive HER 2 expression $(+++)$ measured by immunohistochemistry staining (IHC) underwent gastrectomy at the Affiliated Hospital of the Qingdao University were between March 2013 and December 2017 were retrospectively recruited. Ninety-two stage I-III patients received curative gastrectomy and 6 stage IV patients received palliative gastrectomy. This study was approved by the Ethics Committee of the Affiliated Hospital of Qingdao University and informed consent was obtained from all patients. For each tumor, formalin-fixed, paraffin-embedded (FFPE) tumor tissue was obtained after surgery, $78.6 \%$ samples (77 of 98 ) had a tumor content $\geq 50 \%$ (ranged from 50 to $80 \%$ ) and $21.4 \%$ samples ( 21 of 98 ) were lower than $50 \%$ (ranged from 10 to $40 \%$ ). Disease-free survival (DFS) was defined as the interval from the date of surgery to the date of first locoregional tumor recurrence, distant metastasis or death. Overall survival (OS) was defined as the interval from the date of surgery to the date of death. Patients without an event were censored at the time of the last visit in follow up.

\section{Immunohistochemistry staining and results scoring}

IHC staining was performed on $4 \mu \mathrm{m}$-thick FFPE tissue sections according to the manufacturer's instructions. Briefly, after deparaffinization, heat-induced antigen retrieval and endogenous peroxidase blocking, sections were incubated with ready-to-use anti-HER2 rabbit monoclonal primary antibody (clone 4B5, Ventana, Roche diagnostics, Indianapolis, IN, USA). Staining was visualized using the Ventana ultraView Universal DAB Detection (Ventana, Roche diagnostics, Indianapolis, IN, USA). Slides were lightly counterstained with hematoxylin. Positive and negative controls were included in each assay.

IHC staining results of HER2 expression was scored based on the intensity of membranous staining in tumor cells. HER2 IHC were evaluated according to Hofmann's criteria for gastric cancer (Hofmann et al. 2008), which is scored as 0 (negative), $1+$ (negative), $2+$ (equivocal) or $3+$ (positive). Those samples with a score of $3+$ were considered HER2-positive and were subjected to further capture-based targeted sequencing. IHC scoring was performed by two independent pathologists and a final score was achieved by consensus.

\section{Capture-based targeted sequencing}

FFPE DNA was extracted and fragmented followed by end repair, phosphorylation, $\mathrm{dA}$ addition, and adaptor ligation for library construction. Then, libraries enriched with a targeted next-generation sequencing panel (Burning Rock Biotech Ltd., Guangzhou, China) consisting of 41 gastrointestinal cancer-related genes (as Table $\mathrm{S} 1$ shown) were sequenced on Illumina NextSeq 500 (Illumina, Inc., San Diego, CA, USA) with pair-end reads.

\section{Sequencing data processing and prediction of copy number variations}

The sequencing reads were aligned to the human genome (hg19) using Burrows-Wheeler Aligner 0.7.10. Genome Analysis Toolkit 3.2 (Broad Institute, Cambridge, MA) was used for base quality score recalibration. Mutations were filtered against common single nucleotide polymorphisms found in 1000 Genomes ExAC, dbSNP, ESP6500SI-V2, and ClinVar databases. Variant calling was performed by using VarScan. Variants were annotated with ANNOVAR and SnpEff v3.6. All tumor samples showed adequate coverage, with an average sequencing 
depth of $1280 \times$. Identification of copy number variations (CNVs, amplifications and deletions) was executed as a previous description.

\section{Statistical analysis}

Differences in two-groups were accessed by chi-square test for categorical data and $t$-test for continuous variable. Kaplan-Meier curves were compared by using the log-rank test for survival analyses. Univariate and multivariate analyses with the Cox proportional hazards model were applied. $\mathrm{p}<0.05$ was considered as statistically significant. All statistical analyses were performed in SPSS 22.0 (IBM, Armonk, NY, USA) and R language (version R 3.3.3., https://www.rproject.org/).

\section{Results}

\section{Patients characteristics}

A total of 98 (81 males and 17 females) treatment-naïve resectable Chinese GA patients with HER2 overexpression assessed using IHC with stage I-IV GA were enrolled in our study. The median age of the cohort was 64 years (ranged from 35 to 87 years). There were 12 stage I cases (12.2\%), 33 stage II cases (33.7\%), 47 stage III cases (48.0\%) and 6 stage IV cases $(6.1 \%)$. For histological grade, 3 patients (3.1\%) had well differentiated GA, 41 (41.8\%) had moderately differentiated GA, $53(54.1 \%)$ had poorly differentiated GA and the status of histological grade of the remaining 1 patient (1.0\%) was unknown. According to the Lauren's classification, this cohort had 62 intestinal-type cases (63.3\%), 12 diffuse-type cases (12.2\%), and 24 mixed-type cases (24.5\%). Furthermore, 59 patients $(60.2 \%)$ were positive for perineural invasion, and 39 (39.8\%) were negative. There were 60 patients $(61.2 \%)$ with lymphatic/vascular invasion and 38 (38.2\%) without. Eighty-two patients (83.7\%) had a positive ulcer, 14 patients (14.3\%) had a negative ulcer and the status of ulcer findings in remaining 2 patients $(2.0 \%)$ was unknown. Fifteen patients $(15.3 \%)$ had proximal GA, $40(40.8 \%)$ had middle GA, $40(40.8 \%)$ had distal GA and the status of tumor location in remaining 3 patients $(3.1 \%)$ was unknown. The clinical characteristics of the cohort were summarized in Table 1. In addition, a majority of stage II-IV patients received adjuvant chemotherapy.

\section{Mutation profiling in tumor samples}

Ninety-eight HER2-positive samples assessed using IHC analysis were subjected to targeted sequencing. Collectively, 344 genetic alterations spanning 29 genes were identified, including $186 \mathrm{SNVs}, 25$ rearrangements, 5
Table 1 Clinical baseline of patients with resectable HER2-positive GA

\begin{tabular}{ll}
\hline Characteristics & $n(\%)$ \\
\hline Median age (range), years & $64(35-87)$ \\
Gender & \\
Female & $17(17.3 \%)$ \\
Male & $81(82.7 \%)$ \\
Stage & \\
I & $12(12.2 \%)$ \\
II & $33(33.7 \%)$ \\
III & $47(48.0 \%)$ \\
IV & $6(6.1 \%)$ \\
Histological grade & \\
Well differentiated & $3(3.1 \%)$ \\
Moderately differentiated & $41(41.8 \%)$ \\
Poorly differentiated & $53(54.1 \%)$ \\
Unknown & $1(1.0 \%)$ \\
Lauren's classification & \\
Intestinal type & $62(63.3 \%)$ \\
Diffuse type & $12(12.2 \%)$ \\
Mixed type & $24(24.5 \%)$ \\
Perineural invasion & \\
Positive & \\
Negative & $15(15.3 \%)$ \\
Lymphatic/venous invasion & $40(40.8 \%)$ \\
Positive & $40(40.8 \%)$ \\
Negative & $3(3.1 \%)$ \\
Ulcer findings & $59(60.2 \%)$ \\
Positive & $39(39.8 \%)$ \\
Negative & \\
Unknown & $60(61.2 \%)$ \\
Proxiddimal & $38(38.8 \%)$ \\
Unknown & \\
\hline
\end{tabular}

HER2 human epidermal growth factor receptor 2, GA gastric adenocarcinoma, TNM tumor, node, metastasis

indels and 128 copy number amplifications (CNAs). All patients except 2 had at least one concurrent mutation. The most commonly seen concurrent mutations were occurring in TP53, EGFR and PIK3CA, occurring in $84.7 \%$ $(n=83), 13.3 \%(n=13)$ and $12.2 \%(n=12)$ patients, respectively. Eighteen patients $(18.4 \%)$ had mutations in PIK3CA, AKT1 or PTEN. None of the patients carried $N R A S$ or BRAF mutations. Ninety patients had HER2 amplification defined by NGS, resulting in a concordance of $92 \%$. Ten patients had concurrent HER 2 missense mutations, including 4 with S310X. In addition to HER2 amplification, 16 genes were concomitantly co-amplified. 


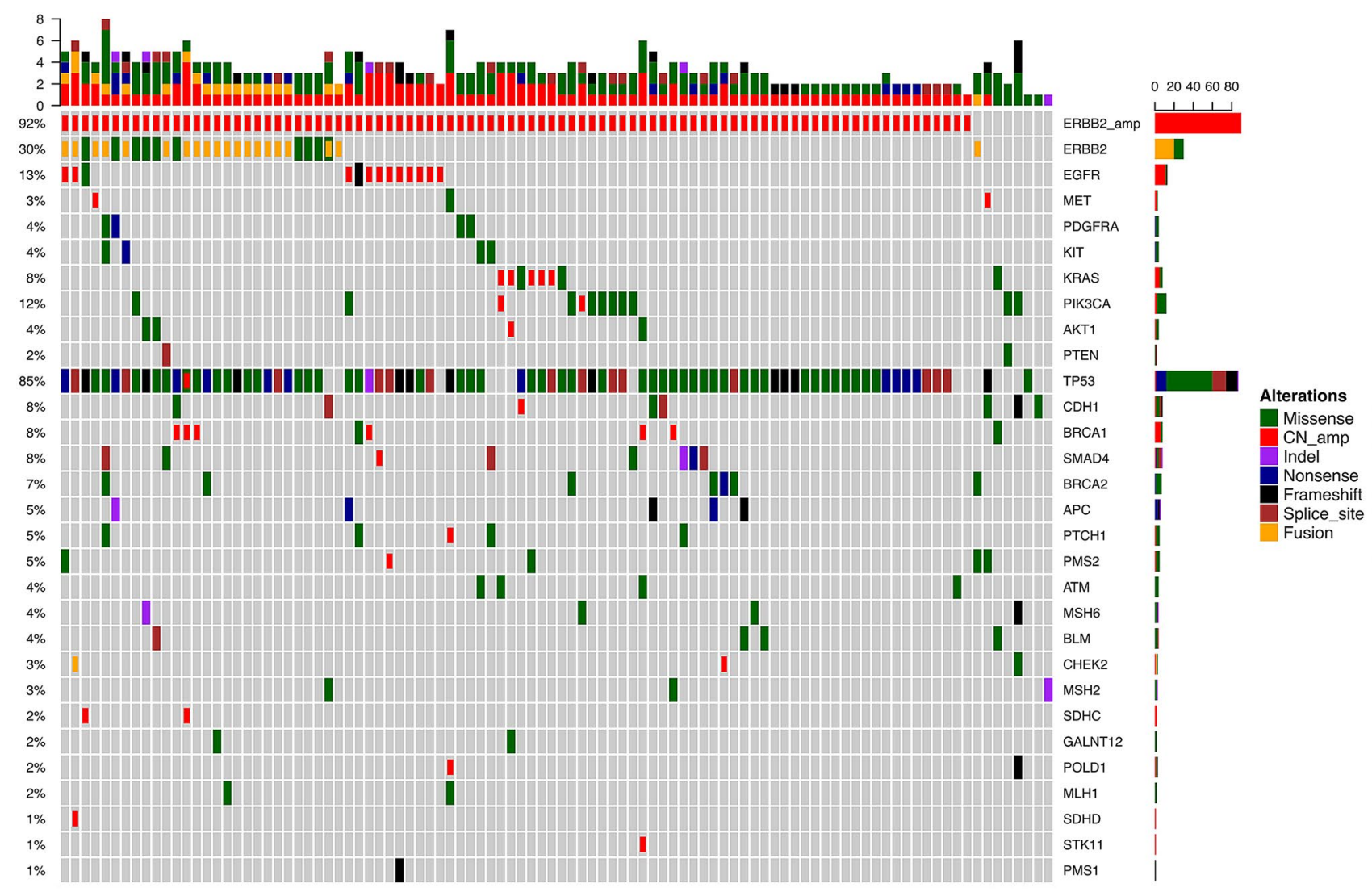

Fig. 1 Heatmap of patients harboring genetic mutations in tumor samples retrieved from targeted next-generation sequencing panel consisting of 41 gastrointestinal cancer-related genes

Six co-amplified genes occurred in more than one patient, including EGFR $(n=11), \operatorname{BRACl}(n=6), \operatorname{KRAS}(n=5)$, PIK3CA $(n=2), \operatorname{SDHC}(n=2), \operatorname{MET}(n=2)$ (Fig. 1).

\section{The association of HER2 copy number with clinicopathologic features}

HER2 copy number (CN) gain was observed in $90 \mathrm{GA}$ patients (90/98) with a median $\mathrm{CN}$ of 15.34 (ranged from 2.67 to 116.27 , Fig. S1), which was used to divide the cohort into $\mathrm{CN}$-high $(n=45)$ and $\mathrm{CN}$-low groups $(n=45)$. Next, we evaluated the association between HER2 CN and several clinical characteristics. Patients with high HER2 CN showed a trend of older compared with patients with low CN ( $p=0.07$, Fig. 2a). According to Lauren's classification, HER2 CN-high group showed a trend of association with intestinal-type GA predominant ( $p=0.075$, Fig. 2d). No significant difference of gender, stage, histological grade, tumor location, perineural invasion, lymphatic/venous invasion and ulcer findings was observed between $\mathrm{CN}$-high and $\mathrm{CN}$-low group (Fig. 2).

\section{The associations between HER2 copy number and survival outcomes}

The associations between HER2 $\mathrm{CN}$ and survival outcomes including DFS and OS in HER2-positive resectable GA patients with stage I-III were evaluated. Forty-nine patients had available $H E R 2 \mathrm{CN}$ and DFS. We investigated whether $H E R 2 \mathrm{CN}$ as a continuous variable was an independent predictor in HER2-positive resectable GA patients. Our work demonstrated that HER $2 \mathrm{CN}$ as a continuous variable was significantly associated with DFS in the univariable Cox proportional hazards regression model (HR: $1.02,95 \% \mathrm{CI}$ $1.00-1.04, p=0.025$ ) and it retained the significant association with DFS in the multivariate Cox proportional hazards regression model (HR: $1.05,95 \%$ CI $1.01-1.09, p=0.029$ ) (Table 2).

Next, the associations between $H E R 2 \mathrm{CN}$ as a categorical variable and survival outcomes in patients were also investigated. Stage distribution in $\mathrm{CN}$ high and low groups was comparable $(p=0.54)$. Our study revealed that DFS of patients with $\mathrm{CN}$-high $(n=26)$ was significantly shorter than that of those with $\mathrm{CN}$-low $(n=23 ; 31$ months vs. not 
A

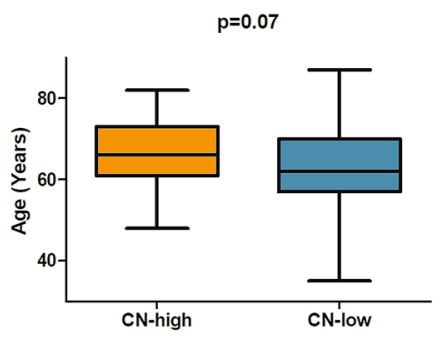

D

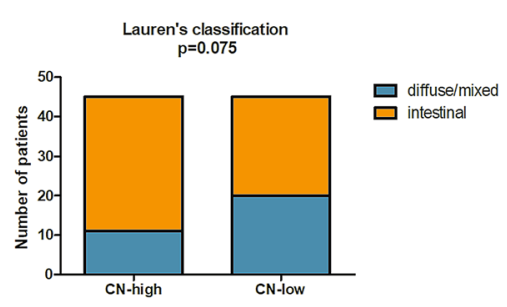

G

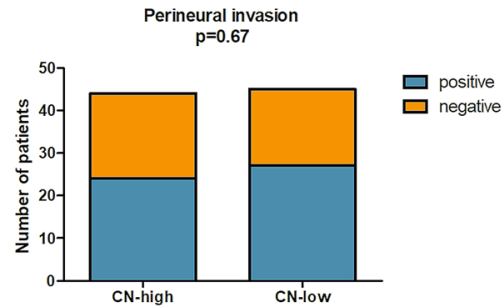

B

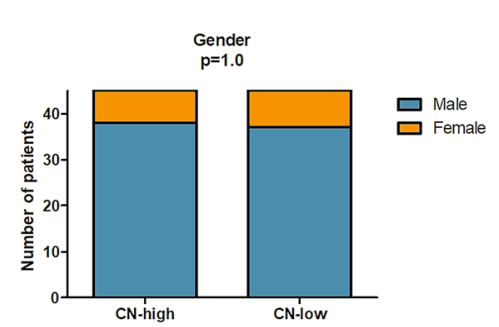

$\mathbf{E}$

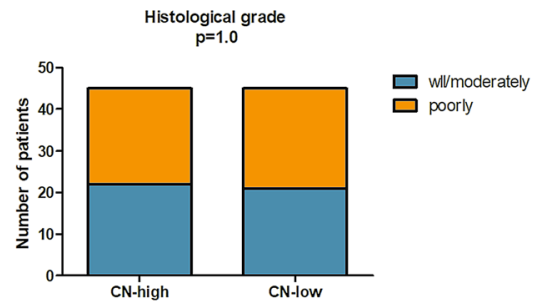

H

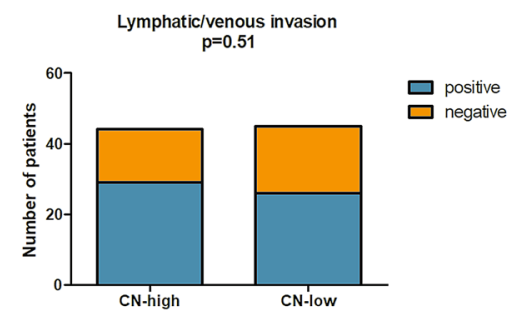

C

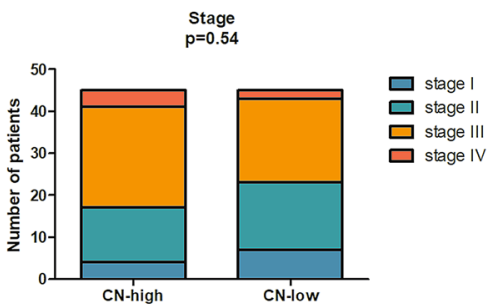

$\mathbf{F}$

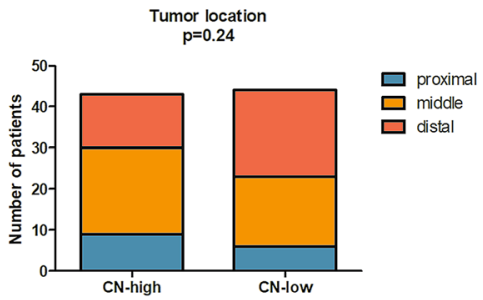

I

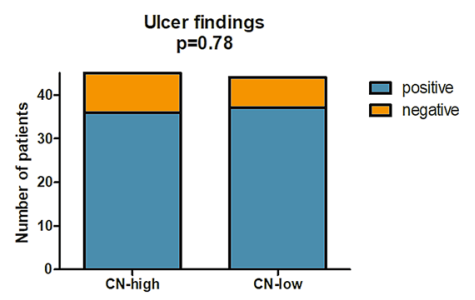

Fig. 2 Correlation of HER2 copy number with clinicopathologic features in HER2-positive resectable GA patients. (a), age; (b), gender; (c), stage; (d), Lauren's classification; (e), histological grade; (f), tumor location; (g), perineural invasion; (h), lymphatic/venous invasion; (i), ulcer findings. GA gastric adenocarcinoma, $C N$ copy number, HER2 human epidermal growth factor receptor 2

Table 2 HER2 CN as a continuous variable was the independent predictor associated with DFS

\begin{tabular}{|c|c|c|c|c|}
\hline \multirow[t]{2}{*}{ Characteristics } & \multicolumn{2}{|c|}{ Univariable analysis } & \multicolumn{2}{|c|}{ Multivariable analysis } \\
\hline & $\mathrm{HR}(95 \% \mathrm{CI})$ & $p$-value & $\overline{\mathrm{HR}(95 \% \mathrm{CI})}$ & $p$-value \\
\hline Gender Male vs. female & $1.47(0.18-11.74)$ & 0.719 & $2.39(0.20-28.96)$ & 0.494 \\
\hline Age $\geq 60$ vs. $<60$ years & $2.81(0.58-13.63)$ & 0.198 & $6.67(0.55-80.91)$ & 0.136 \\
\hline Stage III vs. I/II & $3.59(0.76-16.98)$ & 0.108 & $0.98(0.08-11.79)$ & 0.985 \\
\hline Histological grade poorly vs. well/moderately & $3.18(0.67-15.03)$ & 0.145 & $9.41(0.33-267.23)$ & 0.189 \\
\hline Lauren's classification intestinal vs. diffuse/mixed & $0.62(0.17-2.31)$ & 0.474 & $1.15(0.12-10.72)$ & 0.900 \\
\hline Perineural invasion positive vs. negative & $1.54(0.38-6.15)$ & 0.543 & $0.37(0.05-2.69)$ & 0.326 \\
\hline Lymphatic/venous invasion positive vs. negative & $2.05(0.52-8.07)$ & 0.303 & $7.24(0.84-62.56)$ & 0.072 \\
\hline Ulcer findings positive vs. negative & $2.09(0.26-16.73)$ & 0.487 & $7.60(0.24-239.13)$ & 0.249 \\
\hline Tumor location distal vs. middle/proximal & $0.57(0.12-2.75)$ & 0.483 & $0.78(0.09-6.62)$ & 0.823 \\
\hline$H E R 2 \mathrm{CN}$ & $1.02(1.00-1.04)$ & 0.025 & $1.05(1.01-1.09)$ & 0.029 \\
\hline
\end{tabular}

$D F S$ disease-free survival, $H R$ hazard ratio, $C I$ confidence interval, $C N$ copy number, $H E R 2$ : human epidermal growth factor receptor 2 
A

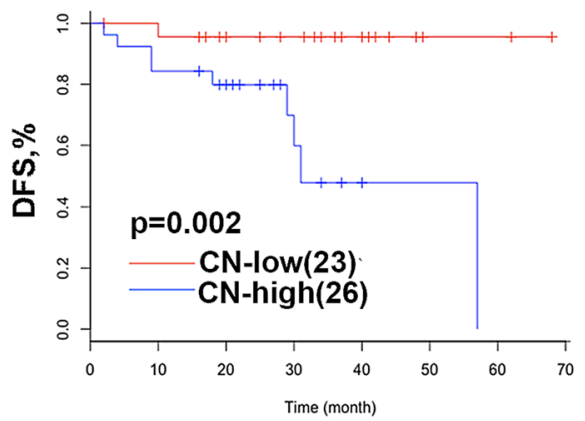

B

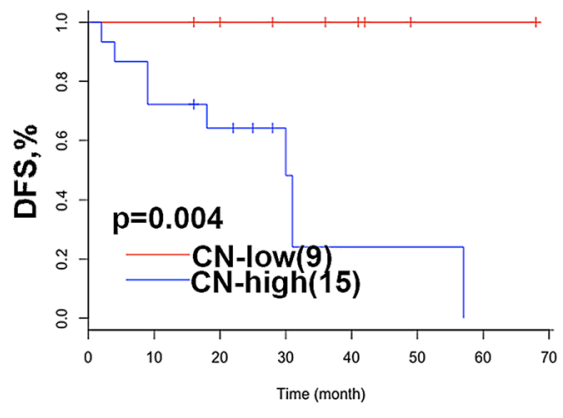

C Stage III patients

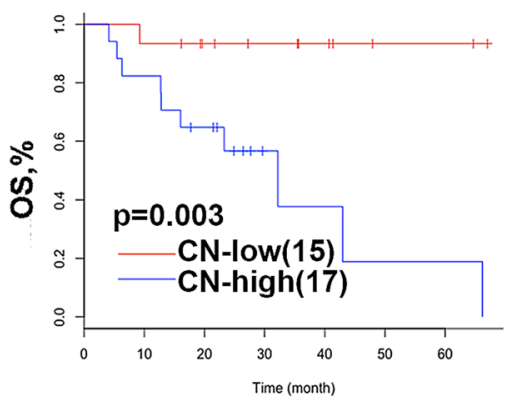

Fig. 3 Kaplan-Meier curves of HER2 $\mathrm{CN}$-high and $\mathrm{CN}$-low patients for DFS and OS. a Kaplan-Meier curves of HER2 CN-high and CNlow patients for DFS in stage I-III patients; $\mathbf{b}$, Kaplan-Meier curves of HER2 CN-high and CN-low patients for DFS in stage III patients; c Kaplan-Meier curves of HER2 CN-high and CN-low patients for OS in stage III patients. DFS disease-free survival, $O S$ overall survival, $C N$ copy number, HER2 human epidermal growth factor receptor 2

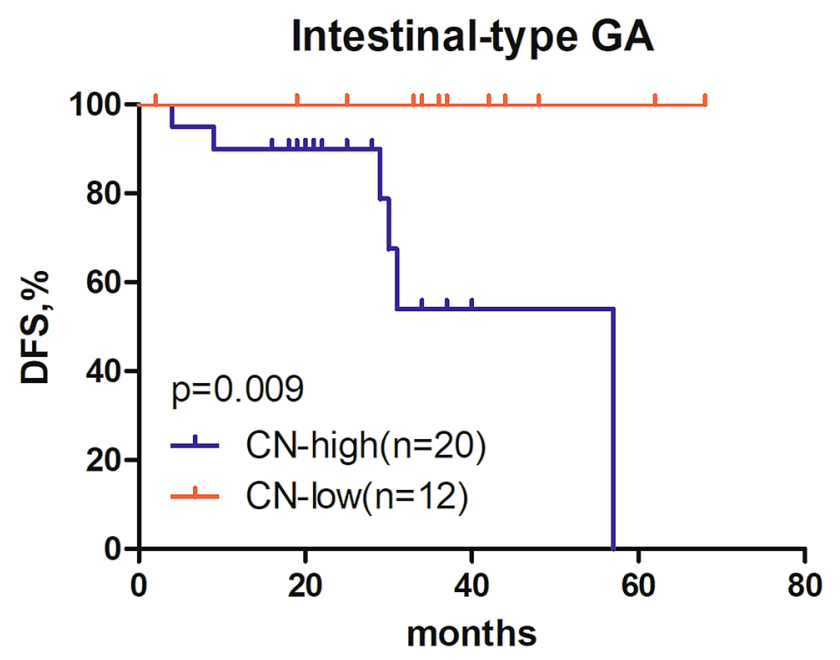

Fig. 4 Kaplan-Meier curves of HER2 CN-high and CN-low patients for DFS in patients with intestinal-type GA. DFS: disease-free survival. CN: copy number; HER2: human epidermal growth factor receptor 2

reached; $p=0.002$; Fig. 3a). A detailed survival analysis was done on patients with stage III disease due to the completeness of survival data. Our observation of shorter DFS in patients with higher CN was confirmed in this subset of patients. Of 24 stage III patients with available DFS, patients with CN-high $(n=15)$ had significantly shorter DFS than those with CN-low ( $n=9 ; 31$ months $v s$. not reached; $p=0.004$; Fig. 3b). Of 32 patients with the available OS, patients with $\mathrm{CN}$-high $(n=17)$ also had significantly shorter OS than those with CN-low ( $n=15 ; 32$ months $v s$. not reached; $p=0.003$; Fig. $3 \mathrm{c}$ ). Furthermore, stratified analyses were performed to explore the correlations of HER2 $\mathrm{CN}$ with survival outcomes in intestinal-type GA patients due to the fact that a majority of patients was diagnosed with intestinal-type GA in our work. Of 32 intestinal-type GA patients with available DFS, patients with CN-high $(n=20)$ had significantly shorter DFS than those with CNlow ( $n=12 ; 57$ months vs. not reached; $p=0.009$; Fig. 4). Collectively, our analyses revealed that patients with high $\mathrm{CN}$ are associated with shorter DFS. Furthermore, stage III patients with high $\mathrm{CN}$ are associated with shorter OS.

In order to evaluate whether $H E R 2 \mathrm{CN}$ as a categorical variable was the independent predictor associated with DFS in HER2-positve resectable GA patients, univariable Cox proportional hazards regression model analysis was performed. In the univariable Cox proportional hazards regression model (Table 2), only HER2 level was associated with DFS (HR: 11.7, 95\% CI 1.45-94.15, $p=0.021$ ). Furthermore, in the multivariate Cox proportional hazards regression model that included gender, age, stage, histological grade, Lauren's classification, perineural invasion, lymphatic/venous invasion, ulcer findings, tumor location and HER2 level, HER2 level retained the significant association with DFS (HR: 32.6, 95\% CI 2.53-419.64, $p=0.008$ ) (Table S2). Collectively, HER2 level either as a continuous variable or as a categorical variable was the independent predictor associated with DFS in HER2-positve resectable GA patients.

\section{Correlations between concomitant alterations and clinical outcomes}

The correlations between concomitant alterations including TP53, EGFR and PIK3CA mutations and clinical outcomes in HER2-positive resectable GA patients were also investigated. HER2 $\mathrm{CN}$ was comparable in patients with and without TP53 mutations, in patients with and without EGFR mutations, in patients with and without PIK3CA mutations (Fig. S2A-S2C). Of 49 GA patients with available DFS, 
DFS of patients with TP53 mutations $(n=46)$ and that of patients without TP53 mutations $(n=3 ; 57$ months vs. not reached; $p=0.42$; Figure S3A) was comparable; DFS of patients with $E G F R$ mutations $(n=7)$ and that of patients without $E F G R$ mutations ( $n=42 ; 57$ months vs. not reached; $p=0.29$; Figure S3B) was comparable; DFS of patients with PIK3CA mutations $(n=6)$ and that of patients without PIK3CA mutations ( $n=43 ; 57$ months vs. not reached; $p=0.66$; Fig. S3C) was also comparable. Collectively, our analyses revealed that concurrent mutations in TP53, $P I 3 K C A$ and EGFR had no impact on DFS.

\section{Discussion}

The predictive value of HER2/HER2 levels in HER2 inhibitor-treated advanced GA patients has been investigated. However, its predictive value in resectable patients remains controversial. In this study, NGS was used to evaluate HER2 $\mathrm{CN}$ in HER2-positve resectable GA patients. Our work revealed that $H E R 2 \mathrm{CN}$ either as a categorical variable or as a continuous variable was the independent risk factor associated with DFS in HER2-positve resectable GA patients.

NGS has become a useful tool for genomic profiling. We confirmed the performance in evaluating CNV using NGS, achieving a concordance of $92 \%$ between NGS and IHC for evaluating HER2 CN in GA tumor samples, which were consistent with previous studies (Cenaj et al. 2019; Pfarr et al. 2017; Ross et al. 2017; Su et al. 2017; Yeh et al. 2019). In our work, median HER $2 \mathrm{CN}$ accessed using NGS was 15.34 (ranged from 2.67 to 116.27) in HER2-positive resectable GA patients with a majority of them (94.4\%) having a HER2 $\mathrm{CN}$ less than 50, which was in an agreement with previous studies that median HER2 $\mathrm{CN}$ accessed by FISH is 11.9 (ranged from 3.30 to 43.80) and it accessed by droplet digital polymerase chain reaction is about 14 (ranged from 3 to 50) in HER2-positive GC patients (Gomez-Martin et al. 2013; Kim et al. 2020). Our study revealed that intestinal-type GA is more likely to harbor a high HER2 $\mathrm{CN}(p=0.075)$, which was consistent with the previous findings that HER2 overexpression is associated with intestinal-type GC (Kataoka et al. 2013; Kim et al. 2019; Kurokawa et al. 2015).

Previous reports revealed that HER2 overexpression or gene amplification correlated with unfavorable prognosis in resectable GA patients (Kurokawa et al. 2015; Otsu et al. 2015). A similar result was observed in our study that HER2 CN-high predicted shorter DFS in patients with HER2-positive resectable GA. Although our and prior studies reported that HER 2 overexpression led to a poor outcome; in contrast, others reported that HER2 expression level was not related to prognosis in resectable GC (Kataoka et al. 2013; Kim et al. 2019; Shen et al. 2016). The controversial results may be attributed to several factors. First, different methods for assessing HER2/HER2 level were used across studies, including but not limited IHC, fluorescence in situ hybridization (FISH) and NGS. IHC and FISH constitute the current gold standard for HER2 assessment. Tumor samples with HER2 IHC $3+$ or IHC $2+/$ FISH + are identified as HER2-positive expression (Hofmann et al. 2008; Muller et al. 2015). There are limitations associated with IHC and FISH for evaluating HER2 overexpression or HER2 amplification, including variation among different antibody sensitivities and specificities, interobserver variabilities (Koopman et al. 2018; Layfield et al. 2016; Sheffield et al. 2014). Second, different studies included different study population. For example, HER2/HER2 status is not correlated with prognosis in a cohort consisting of stage I-IV resectable GC patients; however, a negative correlation is apparent in stage III/IV patients (Kataoka et al. 2013). Third, tumor heterogeneity is more frequently seen in GC (Hofmann et al. 2008). The discordance of HER 2 overexpression between different areas of the same primary tumor occurred in $14.5 \%$ of GC patients, which may lead to an inconsistent assessment of HER2 status (Kim et al. 2011). In addition, the median HER2 CN of 15.34 as the cutoff for predicting longer DFS was identified in our work. Evidence suggest that HER2 $\mathrm{CN}$ of 10 and 9.5 is the optimal cutoff for predicting OS longer than 12 months and for OS longer than 16 months in HER2-positive advanced GC patients treated with trastuzumab, respectively (Gomez-Martin et al. 2013). The cutoff value of HER2 CN or predicting longer DFS in HER2-positive resectable GA patients needed to be validated in larger cohorts.

The incidence rate of GC remains high in East Asian countries, primarily attributing to the high prevalence of Helicobacter pylori (H. pylori) infection; in contrast, it is relatively rare in Western countries (Ferlay et al. 2013; Sugano 2019). Previous study reveals that $H$. pylori infection inducing the aberrant activation-induced cytidine deaminase (AID) via NF- $\mathrm{KB}$ activation, results in mutation accumulation in the gastric mucosa during $H$. pylori-associated gastric carcinogenesis (Maeda et al. 2017; Matsumoto et al. 2007). Yoo and colleagues have reported that HER2 overexpression is more prevalent in $H$. pylori-positive GC (Yoo et al. 2014). The abovementioned findings prompt us to speculate that the HER2/HER2 levels might be different between East Asian and Western population. Several studies have reported the correlation between prognosis and HER2/HER2 levels in East Asian population (Kataoka et al. 2013; Kim et al. 2019; Kurokawa et al. 2015; Shen et al. 2016); however, the prognostic value of HER2/HER2 levels in Western patients with resectable GC remain elusive. To the best of our knowledge, only one study has documented the lack of correlation between HER2 expression level and prognosis in American patients with resectable GC (Fisher et al. 2014). The 
prognostic value of HER2/HER2 levels in Western population needed to be further investigated.

In our work, TP53, EGFR and PIK3CA as classic driver genes (Ge et al. 2017) for gastric cancer, were the most commonly seen concomitant alterations in HER2-positive resectable GA patients. We found the presence of TP53/ EGFR/PIK3CA mutations was not correlated with survival outcomes in HER2-positive resectable GA patients. The previous study also demonstrates that TP53 mutations and EGFR amplification do not have an impact on progressionfree survival of HER2-positive GC patients treated with trastuzumab (Lee et al. 2015). Furthermore, Harada et al. has reported PIK3CA mutations are not associated with DFS nor OS in patients with GC (Harada et al. 2016).

There are several limitations associated with our work. First, the prognostic value of HER2 CN in HER2-positive resectable GA patients should be validated in a large prospective cohort. Second, intratumoral HER2/HER2 heterogeneity was not evaluated in our study, which might result in bias of accurate assessment of HER2/HER 2 status due to the fact that intratumoral HER2/HER2 heterogeneity is particularly significant in GC, ranging from 6 to $69 \%$ of HER2-postive GC patients and predicts unfavorable prognosis in HER2-positive GC (Grillo et al. 2016; Kaito et al. 2019). Further research is still needed to clarify the relevance of intratumoral HER2/HER2 heterogeneity for the survival outcomes in HER2-positive resectable GA patients with HER2 CN-high or CN-low.

In this study, the correlations between $H E R 2 \mathrm{CN}$ and survival outcomes in HER2-positive resectable GA patients were investigated. Our findings demonstrated that HER2 level was the independent predictor associated with DFS in HER2-positve resectable GA patients. Our work indicated $H E R 2$ level can serve as a biomarker in predicting prognosis of patients with HER2-positive resectable GA.

Author contributions ZL: Conceptualization, Methodology, Supervision, Project administration; MS: Methodology, Data Curation, Resources; XL: Methodology, Data Curation, Resources; SS: Methodology, Data Curation, Resources; NL: Methodology, Resources; HD: Writing-Original Draft, Data Curation; JY: Formal analysis, Data Curation; ZZ: Formal analysis, Data Curation; LZ: Data analysis, Data Curation; HL: Data Curation. All authors approved the final manuscript.

Funding The project was supported by the Beijing Xisike Clinical Oncology Research Foundation [Grant Numbers Y-MX2016-004].

\section{Compliance with ethical standards}

Conflict of interest The authors made no disclosures.

Ethical approval Institutional review board approval was obtained from the Ethics Committee of The Affiliated Hospital of Qingdao University.
Consent to participate Informed consent was obtained from each patient for the use of their tumor tissue samples.

Open Access This article is licensed under a Creative Commons Attribution 4.0 International License, which permits use, sharing, adaptation, distribution and reproduction in any medium or format, as long as you give appropriate credit to the original author(s) and the source, provide a link to the Creative Commons licence, and indicate if changes were made. The images or other third party material in this article are included in the article's Creative Commons licence, unless indicated otherwise in a credit line to the material. If material is not included in the article's Creative Commons licence and your intended use is not permitted by statutory regulation or exceeds the permitted use, you will need to obtain permission directly from the copyright holder. To view a copy of this licence, visit http://creativecommons.org/licenses/by/4.0/.

\section{References}

Bang YJ et al (2010) Trastuzumab in combination with chemotherapy versus chemotherapy alone for treatment of HER2-positive advanced gastric or gastro-oesophageal junction cancer (ToGA): a phase 3, open-label, randomised controlled trial. Lancet 376:687697. https://doi.org/10.1016/s0140-6736(10)61121-x

Bray F, Ferlay J, Soerjomataram I, Siegel RL, Torre LA, Jemal A (2018) Global cancer statistics 2018: GLOBOCAN estimates of incidence and mortality worldwide for 36 cancers in 185 countries. CA Cancer J Clin 68:394-424. https://doi.org/10.3322/ caac. 21492

Cenaj O, Ligon AH, Hornick JL, Sholl LM (2019) Detection of ERBB2 amplification by next-generation sequencing predicts HER2 expression in colorectal carcinoma. Am J Clin Pathol 152:97-108. https://doi.org/10.1093/ajcp/aqz031

Cordero-García E et al (2019) Evaluation of the expression of the oncogen C-ERBB-2/HER2 in advanced gastric cancer cases from Costa Rica. Ecancermedicalscience 13:962. https://doi. org/10.3332/ecancer.2019.962

Coussens L et al (1985) Tyrosine kinase receptor with extensive homology to EGF receptor shares chromosomal location with neu oncogene. Science (New York, NY) 230:1132-1139. https:// doi.org/10.1126/science. 2999974

Ferlay J et al (1990) (2013) Cancer incidence and mortality patterns in Europe: estimates for 40 countries in 2012. Eur J Cancer England 49:1374-1403. https://doi.org/10.1016/j.ejca.2012.12.027

Fisher SB et al (2014) HER2 in resected gastric cancer: is there prognostic value? J Surg Oncol 109:61-66. https://doi.org/10.1002/ jso. 23456

Garcia I, Vizoso F, Martin A, Sanz L, Abdel-Lah O, Raigoso P, Garcia-Muniz JL (2003) Clinical significance of the epidermal growth factor receptor and HER2 receptor in resectable gastric cancer. Ann Surg Oncol 10:234-241. https://doi.org/10.1245/ aso.2003.05.010

Ge S et al (2017) Genomic alterations in advanced gastric cancer endoscopic biopsy samples using targeted next-generation sequencing. Am J Cancer Res 7:1540-1553

Gomez-Martin C et al (2013) Level of HER2 gene amplification predicts response and overall survival in HER2-positive advanced gastric cancer treated with trastuzumab. J Clin Oncol 31:44454452. https://doi.org/10.1200/jco.2013.48.9070

Grillo F, Fassan M, Sarocchi F, Fiocca R, Mastracci L (2016) HER2 heterogeneity in gastric/gastroesophageal cancers: from benchside to practice World. J Gastroenterol 22:5879-5887 
Harada K et al (2016) Prognostic and clinical impact of PIK3CA mutation in gastric cancer: pyrosequencing technology and literature review. BMC Cancer 16:400. https://doi.org/10.1186/s1288 5-016-2422-y

Hofmann $M$ et al (2008) Assessment of a HER2 scoring system for gastric cancer: results from a validation study. Histopathology 52:797-805. https://doi.org/10.1111/j.1365-2559.2008.03028.x

Hohenberger P, Gretschel S (2003) Gastric cancer. Lancet 362:305315. https://doi.org/10.1016/s0140-6736(03)13975-x

Hsieh AC, Moasser MM (2007) Targeting HER proteins in cancer therapy and the role of the non-target HER3. Br J Cancer 97:453-457. https://doi.org/10.1038/sj.bjc.6603910

Hynes NE, Stern DF (1994) The biology of erbB-2/neu/HER-2 and its role in cancer. Biochim Biophys Acta 1198:165-184. https://doi. org/10.1016/0304-419x(94)90012-4

Kaito A, Kuwata T, Tokunaga M, Shitara K, Sato R, Akimoto T, Kinoshita T (2019) HER2 heterogeneity is a poor prognosticator for HER2-positive gastric cancer. World J Clin Cases 7:19641977. https://doi.org/10.12998/wjcc.v7.i15.1964

Kataoka Y, Okabe H, Yoshizawa A, Minamiguchi S, Yoshimura K, Haga H, Sakai Y (2013) HER2 expression and its clinicopathological features in resectable gastric cancer. Gastric Cancer 16:84-93. https://doi.org/10.1007/s10120-012-0150-9

Kim B et al (2020) Comparative analysis of HER2 copy number between plasma and tissue samples in gastric cancer using droplet digital PCR. Sci Rep 10:4177. https://doi.org/10.1038/s4159 8-020-60897-4

Kim H et al (2019) Prognostic significance of Human epidermal growth factor receptor-2 expression in patients with resectable gastric adenocarcinoma. World J Surg Oncol 17:122. https://doi. org/10.1186/s12957-019-1652-2

Kim MA, Lee HJ, Yang HK, Bang YJ, Kim WH (2011) Heterogeneous amplification of ERBB2 in primary lesions is responsible for the discordant ERBB2 status of primary and metastatic lesions in gastric carcinoma. Histopathology 59:822-831. https://doi.org/1 0.1111/j.1365-2559.2011.04012.x

King CR, Kraus MH, Aaronson SA (1985) Amplification of a novel v-erbB-related gene in a human mammary carcinoma. Science (New York, NY) 229:974-976. https://doi.org/10.1126/scien ce. 2992089

Koopman T et al (2018) HER2 immunohistochemistry in endometrial and ovarian clear cell carcinoma: discordance between antibodies and with in-situ hybridisation. Histopathology 73:852-863. https ://doi.org/10.1111/his.13704

Kurokawa $\mathrm{Y}$ et al (2015) Multicenter large-scale study of prognostic impact of HER2 expression in patients with resectable gastric cancer. Gastric Cancer 18:691-697. https://doi.org/10.1007/s1012 0-014-0430-7

Layfield LJ, Frazier S, Esebua M, Schmidt RL (2016) Interobserver reproducibility for HER2/neu immunohistochemistry: a comparison of reproducibility for the HercepTest ${ }^{\mathrm{TM}}$ and the 4B5 antibody clone. Pathol Res Pract 212:190-195. https://doi.org/10.1016/j. prp.2015.11.016

Lee JY, Hong M, Kim ST, Park SH, Kang WK, Kim KM, Lee J (2015) The impact of concomitant genomic alterations on treatment outcome for trastuzumab therapy in HER2-positive gastric cancer. Sci Rep 5:9289. https://doi.org/10.1038/srep09289

$\mathrm{Li} \mathrm{H}$ et al (2018) Association between PIK3CA alteration and prognosis of gastric cancer patients: a meta-analysis. Oncotarget 9:76517659. https://doi.org/10.18632/oncotarget.23871

Li YS et al (2018) Unique genetic profiles from cerebrospinal fluid cell-free DNA in leptomeningeal metastases of EGFR-mutant non-small-cell lung cancer: a new medium of liquid biopsy. Ann Oncol 29:945-952. https://doi.org/10.1093/annonc/mdy009
Ma J, Lyu H, Huang J, Liu B (2014) Targeting of erbB3 receptor to overcome resistance in cancer treatment. Mol Cancer 13:105. https://doi.org/10.1186/1476-4598-13-105

Maeda M, Moro H, Ushijima T (2017) Mechanisms for the induction of gastric cancer by Helicobacter pylori infection: aberrant DNA methylation pathway. Gastric Cancer 20:8-15. https://doi. org/10.1007/s10120-016-0650-0

Matsumoto $Y$ et al (2007) Helicobacter pylori infection triggers aberrant expression of activation-induced cytidine deaminase in gastric epithelium. Nat Med 13:470-476. https://doi.org/10.1038/ nm1566

Mizutani T, Onda M, Tokunaga A, Yamanaka N, Sugisaki Y (1993) Relationship of C-erbB-2 protein expression and gene amplification to invasion and metastasis in human gastric cancer. Cancer 72:2083-2088

Moasser MM (2007) The oncogene HER2: its signaling and transforming functions and its role in human cancer pathogenesis. Oncogene 26:6469-6487. https://doi.org/10.1038/sj.onc.1210477

Muller KE, Marotti JD, Memoli VA, Wells WA, Tafe LJ (2015) Impact of the 2013 ASCO/CAP HER2 guideline updates at an Academic Medical Center that performs primary HER2 FISH testing: increase in equivocal results and utility of reflex immunohistochemistry. Am J Clin Pathol 144:247-252. https://doi. org/10.1309/ajcpe5nchwpsmr5d

Otsu H et al (2015) Correlation of HER2 expression with clinicopathological characteristics and prognosis in resectable gastric cancer. Anticancer Res 35:2441-2446

Pfarr N et al (2017) Targeted next-generation sequencing enables reliable detection of HER2 (ERBB2) status in breast cancer and provides ancillary information of clinical relevance. Genes Chromosomes Cancer 56:255-265. https://doi.org/10.1002/gcc.22431

Qiu MZ et al (2017) Comparison of HER2 and Lauren Classification between Biopsy and Surgical Resection Samples, Primary and Metastatic Samples of Gastric Cancer. J Cancer 8(17):3531-3537. https://doi.org/10.7150/jca.19984

Ross DS et al (2017) Next-generation assessment of human epidermal growth factor receptor 2 (ERBB2) amplification status: clinical validation in the context of a hybrid capture-based comprehensive solid tumor genomic profiling assay. J Mol Diagn 19:244-254. https://doi.org/10.1016/j.jmoldx.2016.09.010

Sheffield BS, Garratt J, Kalloger SE, Li-Chang HH, Torlakovic EE, Gilks CB, Schaeffer DF (2014) HER2/neu testing in gastric cancer by immunohistochemistry: assessment of interlaboratory variation. Arch Pathol Laborat Med 138:1495-1502. https://doi. org/10.5858/arpa.2013-0604-OA

Shen GS et al (2016) Association of HER2 status with prognosis in gastric cancer patients undergoing R0 resection: a large-scale multicenter study in China. World J Gastroenterol 22:5406-5414. https://doi.org/10.3748/wjg.v22.i23.5406

Shitara K et al (2013) Prognosis of patients with advanced gastric cancer by HER 2 status and trastuzumab treatment. Gastric Cancer 16:261-267. https://doi.org/10.1007/s10120-012-0179-9

Siegel RL, Miller KD, Jemal A (2020) Cancer statistics, 2020. CA Cancer J Clin 70:7-30. https://doi.org/10.3322/caac.21590

Su D et al (2017) High performance of targeted next generation sequencing on variance detection in clinical tumor specimens in comparison with current conventional methods . J Exp Clin Cancer Res 36:121. https://doi.org/10.1186/s13046-017-0591-4

Sugano K (2019) Effect of Helicobacter pylori eradication on the incidence of gastric cancer: a systematic review and meta-analysis. Gastric Cancer 22:435-445. https://doi.org/10.1007/s1012 0-018-0876-0

Van Cutsem E, Sagaert X, Topal B, Haustermans K, Prenen H (2016) Gastric cancer Lancet 388:2654-2664. https://doi.org/10.1016/ s0140-6736(16)30354-3 
Yarden Y, Sliwkowski MX (2001) Untangling the ErbB signalling network. Nature reviews. Mol Cell Biol 2:127-137. https://doi. org/10.1038/35052073

Yeh YM et al (2019) Comprehensive assessment of HER2 alteration in a colorectal cancer cohort: from next-generation sequencing to clinical significance. Cancer Manage Res 11:7867-7875. https:// doi.org/10.2147/cmar.s213247

Yoo MW et al (2014) Is helicobacter pylori associated with Her2/neu overexpression in gastric cancer patients who underwent curative resection? Hepatogastroenterology 61:858-862
Publisher's Note Springer Nature remains neutral with regard to jurisdictional claims in published maps and institutional affiliations. 